\title{
Research and application of power grid intelligent inspection management system based on physical ID
}

\author{
*QU Limin, WANG Chao, ZHANG Jian, ZHANG Hang, SUN Wei, SHENG Jie \\ State Grid Heilongjiang Electric Power Company Limited Electric Power Research Institute, Harbin 150030, Heilongiiang Province, \\ China
}

\begin{abstract}
Aiming at the problems of low efficiency, poor accuracy and untimely defect detection of traditional power grid equipment inspection methods, this paper proposed a power grid intelligent inspection management system based on physical ID. And a method for identifying high-risk alarm areas and fault location of transmission channel is proposed. By using big data mining and unsupervised clustering machine learning algorithm, the problems of poor accuracy and slow calculation speed of a large number of alarm data area division are fundamentally solved, and the functions of dynamic alarm and location affected by external force destruction, foreign object intrusion and environment are realized. The results show that compared with the traditional methods, the proposed method has higher efficiency and accuracy, and lower fault trip rate.
\end{abstract}

\section{Introduction}

The contradiction between the grid scale growth and the lack of personnel of operation-maintenance is becoming increasingly prominent. The traditional operation mode of manual copying has many disadvantages, such as producing a large number of paper documents, missing filling and reporting 、 high operation risk, low inspection efficiency, single device function, poor accuracy and intelligence, low reliability of traditional detection equipment in low temperature environment ${ }^{[1-3]}$. Therefore, it is urgent to carry out research on key technologies such as intelligent inspection and monitoring of power generation, transmission, transformation and distribution, so as to improve the work efficiency of front-line operators and the operation and maintenance management level of power equipment ${ }^{[4]}$.

In this article, an intelligent mobile inspection system for electrical generation, transmission, transformation and distribution based on physical ID is developed ${ }^{[5]}$. And the identification method for high occurrence area of visual alarm and fault location technology of transmission channel are studied. The research results improve the inspection efficiency and accuracy of power equipment, and realize the functions of on-line monitoring, intelligent fault identification and early warning.

\section{Software architecture of intelligent inspection system}

\subsection{Overall system architecture}

The intelligent inspection management system is divided into three levels, which from top to bottom is business system layer, mobile application service layer and mobile terminal application layer.

The business system layer includes the main station service of power intelligent inspection, hydropower inspection management service and HPMS master station service. The master station service mainly includes function expansion service, data synchronization service, data analysis service, data buffer service, which provides inspection management of three professions. The hydropower inspection management service mainly provides business functions and data interface services for hydropower inspection management business. Data interaction between hydropower inspection management service and HPMS master station service is realized through interface services, so as to meet the requirements of hydropower inspection management.

The mobile application service layer provides unified interaction between mobile applications and business systems, mainly including identity authentication service, data synchronization service, message push service, mobile data security service, etc. The application layer of mobile terminal provides power intelligent patrol APP and mobile application platform client-side. The mobile application platform client-side provides unified data 
interaction mechanism, unified identity authentication and terminal application management functions.

\subsection{Technology Architecture}

It is designed and implemented with a hierarchical architecture. At runtime, it experiences multiple functional levels of interaction from user interface to data storage, and successively carries out data display and input, data transmission and access, service request distribution, business logic execution and data persistence operation. The technical architecture is shown in Figure 1.
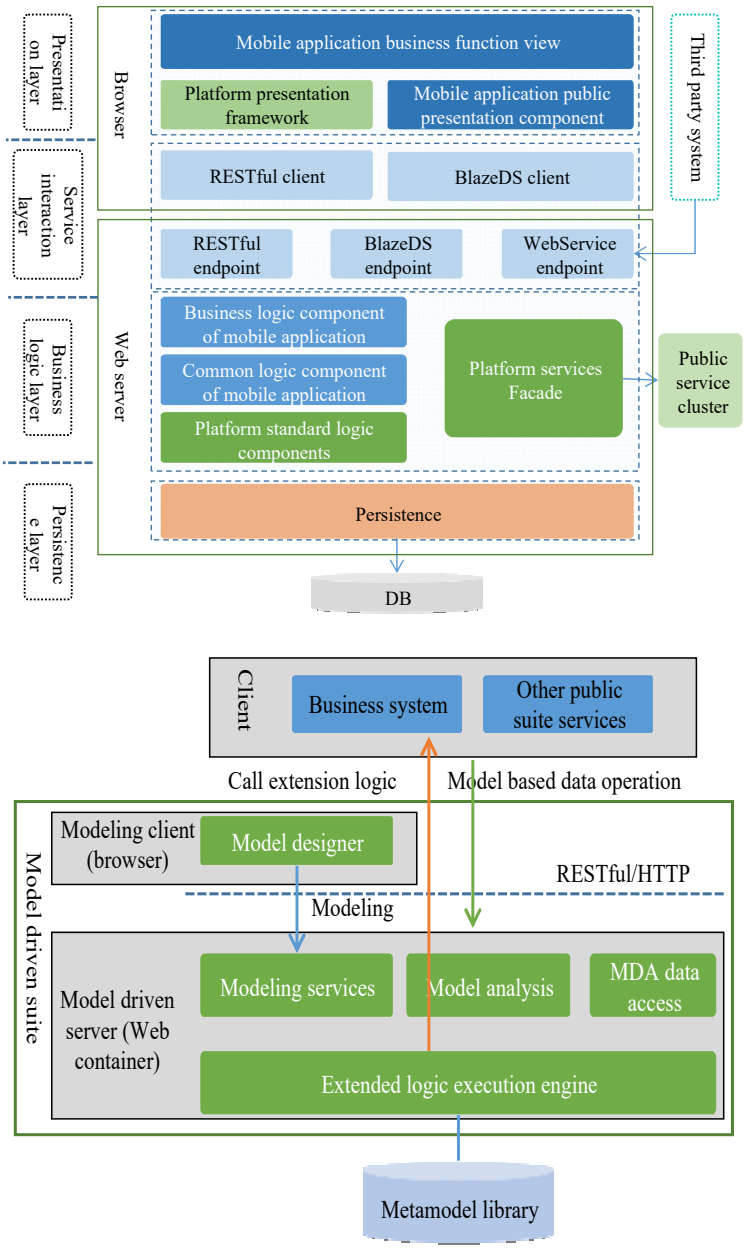

Figure 1. Hierarchical structure of SG-UAP platform technology

The presentation layer includes the platform presentation framework, and the public presentation components and business function pages of mobile terminal application services based on the platform presentation framework. The service interaction layer includes the communication client running in the browser and the service access point running in the server. The business logic layer includes the platform standard logic component, the mobile terminal application service common logic component and the business logic component. The persistence layer provides data persistence and data access capabilities.

\section{Development and research of intelligent patrol function module}

The mobile inspection application systems of power transmission, transformation, distribution and hydropower based on physical ID are developed respectively. The functions of online and offline mode intelligent switching, voice broadcast service based on location information, image recognition, offline voice input and intelligent defect management are realized.

\subsection{Intelligent inspection based on physical ID}

Through the combination of "physical ID + mobile terminal" technology, we can quickly locate the patrol line equipment, timely check the equipment account information, patrol standards and historical defects, quickly register the defects or hidden dangers of the equipment found in the patrol process, and cache the patrol data, improve the patrol record information and automatically generate the next periodic patrol plan. In addition, it can reduce the number of user field operations, improve user efficiency, the defect intelligent management interface is shown in Figure 2.

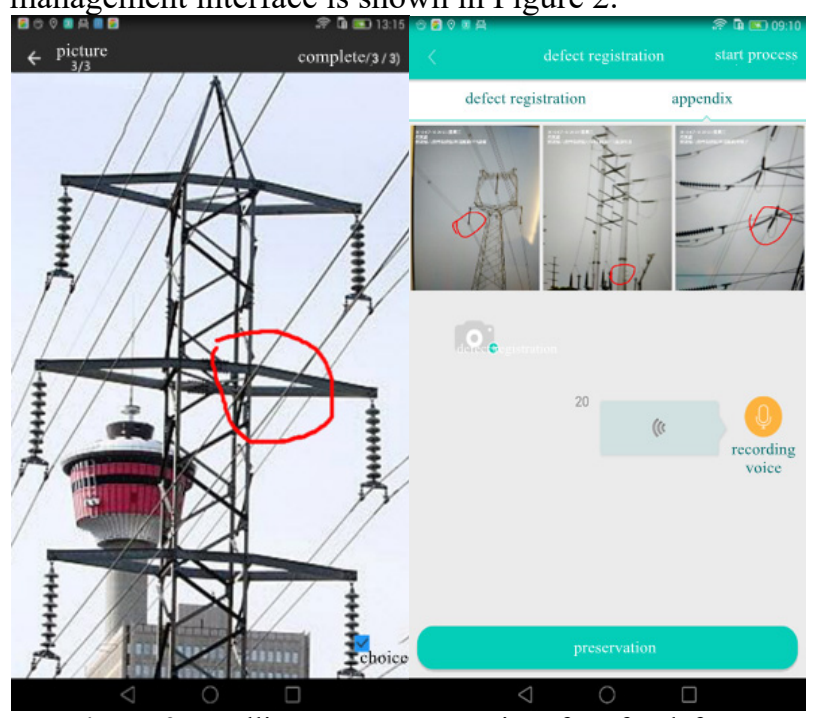

Figure 2. Intelligent management interface for defects

\subsection{Intelligent switching between online and offline modes}

Combined with the actual environment of the work, it can intelligently switch off-line and on-line working modes according to the network signal, and ensure the normal use without network. It can maximize the advantages of the two modes automatically. This function is especially needed when working in urban underground substations and hydropower stations. The online and offline intelligent switching interface is shown in Figure 3. 


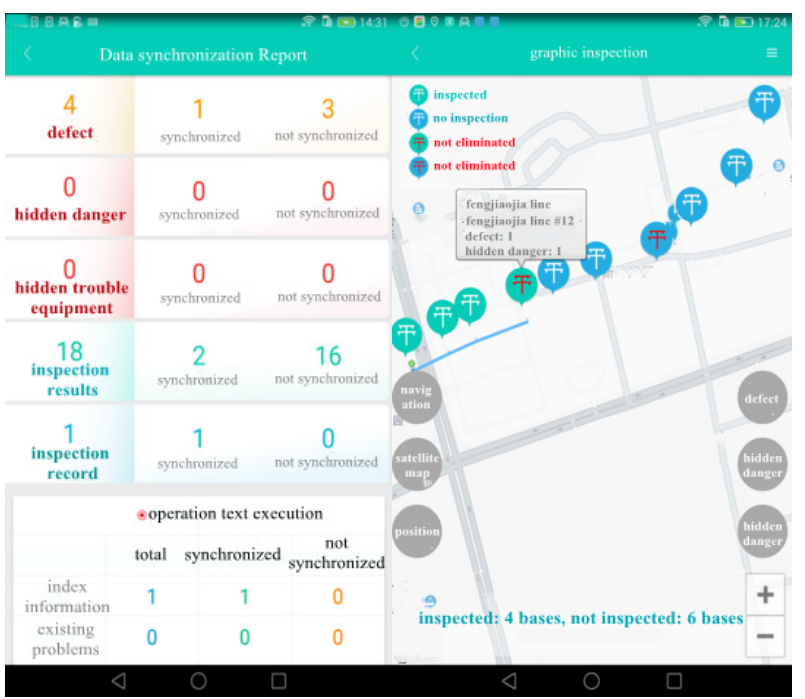

Figure 3. Online and offline intelligent switching interface

\subsection{Voice broadcast service based on location information}

Using real-time offline speech synthesis technology, based on the intranet offline map, the tower position and inspection track can be displayed, where inspectors can navigate to. In the process of inspection, the coordinates are uploaded to PMS2.0 system in real time to provide the basis for inspection quality verification. When defects are found, the nearest tower will be located automatically to simplify the operation steps of the mobile terminal. The interface of voice broadcast and voice input is shown in Figure 4.

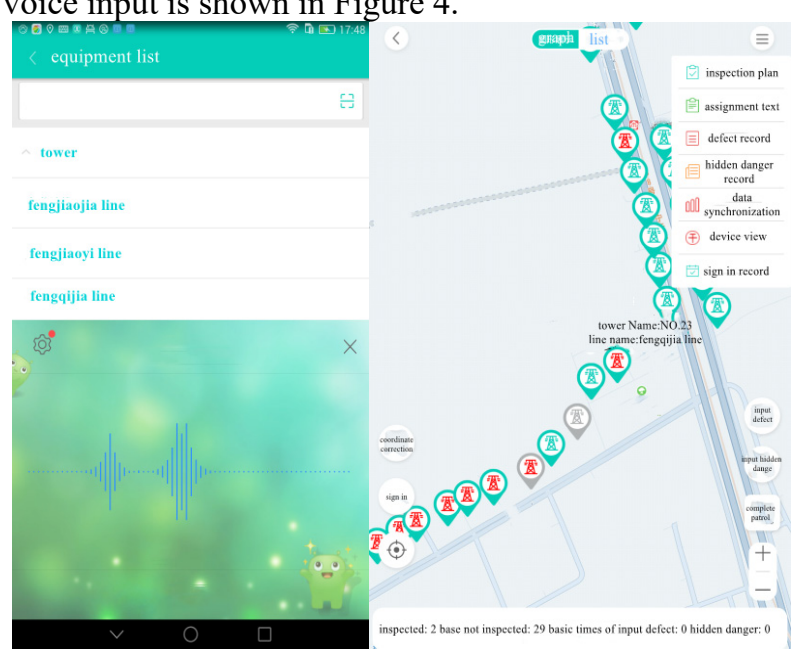

Figure 4. Voice broadcast and voice input interface

\section{Development and research of transmission visualization module}

\subsection{Research on identification method of hidden danger in transmission channel}

Using deep learning algorithm as the basis of scene recognition algorithm, a large number of candidate target area boxes are generated in the image. The depth learning algorithm is used to extract visual features from the image blocks in each candidate frame. The feature extracted from the candidate box is classified into a specific class. For the candidate box belonging to a certain feature, the position of the candidate box is further adjusted by using the regressor, so as to identify the large machinery and other objects that may pose a potential threat to the safety of the transmission line in the scene, and generate early warning information for the relevant personnel to view. Through the collection and edge calculation to analyze the real-time monitoring data, when detected abnormal, timely start the relevant monitoring unit of photography, video, video and other functions, the first time to control the state of the scene. At the same time, the edge IOT agent supports shortrange wireless transmission and wired communication, which can automatically identify channel hidden dangers and body defects, and automatically warn to avoid line trip. The scene of image intelligent recognition is shown in Figure 5.

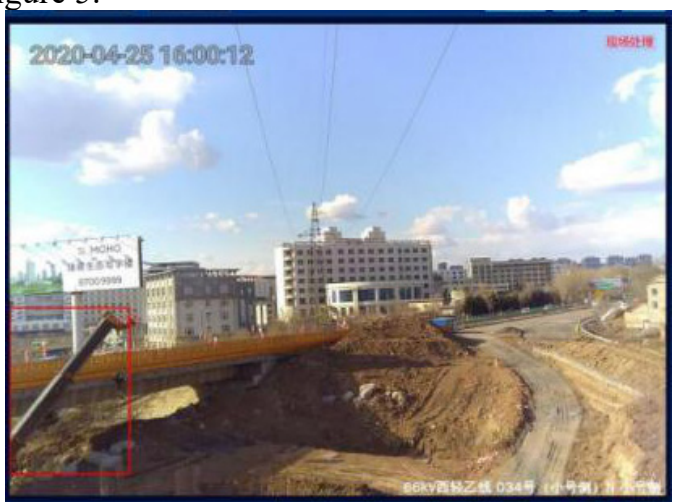

Figure 5. Image intelligent recognition scene display - crane operation under the line

By means of big data analysis and mining, high alarm area identification and high alarm line segment identification are realized. The key hidden danger distribution area within a specified time can be obtained through the identification operation of the high alarm area, which is displayed in a circle on the map. Based on this map, the stationary point inspection can focus on the area with relatively more hidden dangers.

The two principles of identifying the high alarm line segment are that the percentage of the number of alarms in the line segment reaches the specified threshold, and the percentage of the number of towers in the line segment reaches the specified threshold. In order to meet the two requirements, different colors are given to display on the map according to different voltage levels, and through weight marking of alarm data, the unsupervised clustering machine learning algorithm is used to solve the problems of poor accuracy and slow calculation speed of a large number of alarm data area division, and realize the dynamic alarm of external force damage, foreign object intrusion, environmental impact, etc. The process and information display interface are shown in Figure 6. 


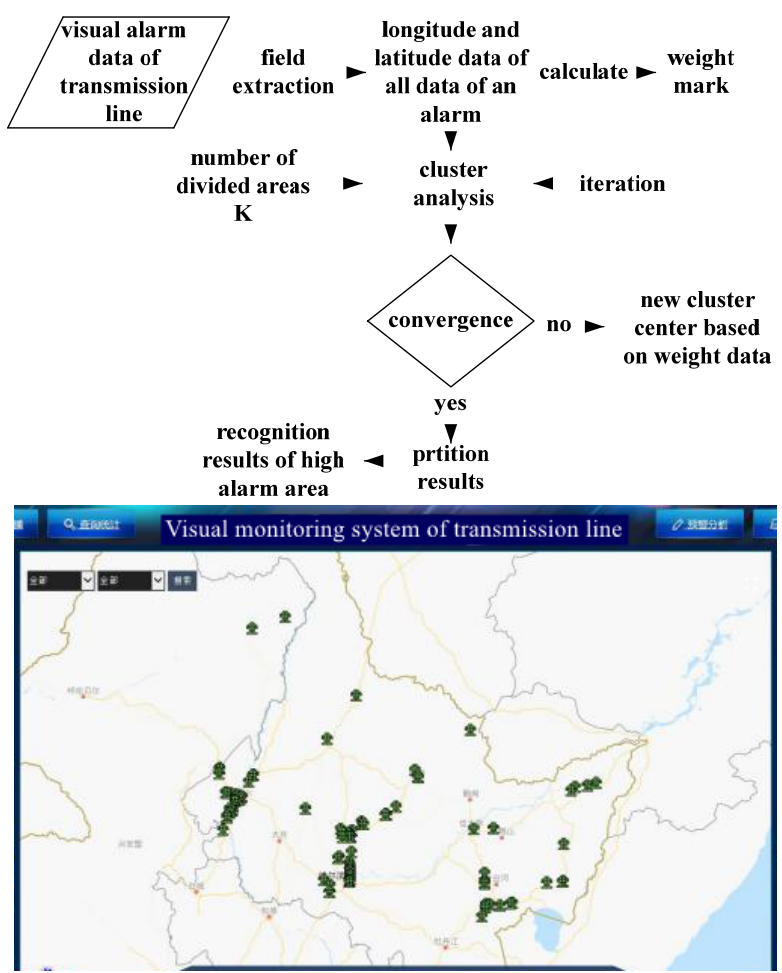

Figure 6. Alarm identification method flow and information display interface

\subsection{Research on hidden danger three- dimensional ranging method}

Using monocular vision based spatial distance analysis technology, by judging the distance between the hidden danger and the transmission body and the high-risk early warning area offline, the hidden danger is divided into different early warning levels, so as to deal with the existing hidden danger in time according to the early warning level. Taking the conductor distance measurement as an example, the distance between the hidden danger and the transmission body is judged, that is, the distance between the hidden danger and the lower phase conductor is regarded as the shortest distance between the hidden danger and the conductor. Furthermore, the pixel distance and the real distance are converted to obtain the real distance between the hidden danger and the power transmission body. The hidden danger distance measurement error based on 3D point cloud data can be controlled at about one meter.

The 3D ranging system measures the distance from the hidden danger to the wire according to the hidden danger coordinates. According to the measurement results, the distance line, hidden danger frame, the real distance from the hidden danger to the wire, the hidden danger height and the protection zone are drawn in the figure, as shown in Figure 7.

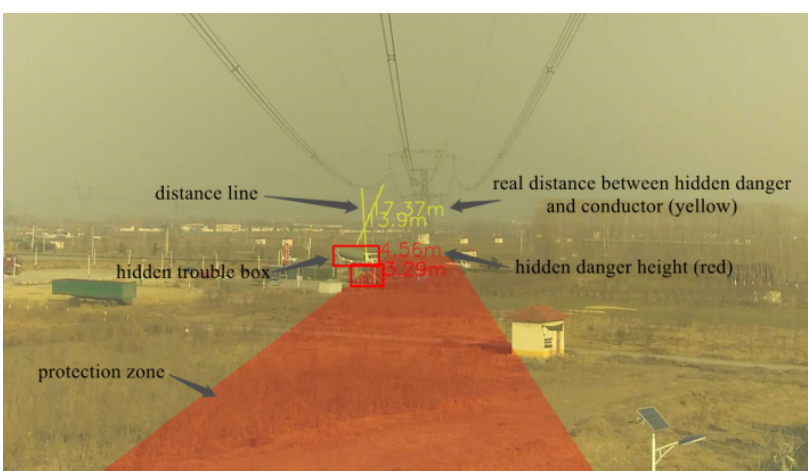

Figure 7. Range recognition effect figure chart

The distance line is the shortest path from the wire to the hidden danger, the hidden danger box is the area calibrated according to the coordinates of the image analysis results, the real distance from the hidden danger to the wire is the shortest path distance measured by the three-dimensional distance measurement service, the hidden danger height is the height measured by the three-dimensional distance measurement service, and the protection area is the protection area from the wire to the ground.

\section{Application effect}

It can comprehensively improve the work efficiency of grass-roots work and reduce the burden of front-line staff. Through on-site code scanning to carry out equipment and financial inventory, the efficiency of substation equipment verification is increased by 2.5 times. Through code scanning to create equipment account, the efficiency of account creation is increased by 2 times. The use of mobile inspection micro application can reduce paper records of all substation specialties, such as 8 kinds of transmission specialties and 8 kinds of distribution specialties, a total of 144 kinds of paper records are reduced, and the average load of teams is reduced by $88.89 \%$, the maintenance accuracy rate was increased by $47 \%$.

The identification method of high frequency area of visual alarm of transmission line channel is proposed for the first time. The dynamic alarm of external force damage, foreign invasion and environmental impact is realized. The identification accuracy is as high as $95 \%$. The line visual monitoring platform of the transmission is built to solve the problem of real-time monitoring of transmission line operation, and the fault trip out rate is reduced by $86.59 \%$.

\section{Conclusion}

Aiming at the problems of low efficiency and poor accuracy of traditional inspection methods for transmission, transformation, distribution and hydropower specialty, this paper develops an intelligent inspection management system for power grid based on physical ID. In addition, the identification method and fault location technology of high occurrence area of visual alarm in transmission channel are deeply studied. The research results improve the inspection efficiency 
and accuracy of power equipment, and realize the functions of online monitoring and intelligent fault identification, early warning and accurate positioning, and ensure the safe and stable operation of power grid and equipment.

\section{References}

1. TAO M J, WENG L J, JIANG X F, et al. (2021) Application of intelligent wearable devices in substation inspection and decision support. Scientific and Technological Innovation, 2021(2): 109-110.

2. ZHANG J, ZHANG Z F, ZHANG L N. (2020) Application and prospect of intelligent inspection technology for power transmission and transformation. China Plant Engineering, 2020(20): 160-162.

3. ZHOU Z Y, CHEN Y P, PAN C, et al. (2020) High reliability and low delay mobile edge computing technology for intelligent power inspection. High Voltage Engineering, 46(6): 1895-1902.

4. RUAN G H, LI W H. (2021) Remote intelligent inspection method of transmission line based on video linkage technology. Automation and Instrumentation, 2021(1): 77-80.

5. QU L M, ZHANG H K, SUN W, et al. (2020) Construction and application of assets unified identity code in Heilongjiang power grid. Heilongjiang Electric Power, 42(5): 385-392. 